Gut, 1961, 2, 360

\title{
Experiences with extraperitoneal colostomy and ileostomy
}

\author{
A. ELLIOT-SMITH AND NEIL S. PAINTER \\ From the Radcliffe Infirmary, Oxford
}

SYNOPSIS A technique for extraperitoneal colostomy and ileostomy has been used by one of the authors since 1952. The technique is described and the results reviewed.

The small intestine may become obstructed after colostomy or ileostomy, due to its prolapsing through the lateral space, and, to avert this possibility, it is a common practice to close the space by suturing the terminal bowel to the parietal peritoneum. Sames (1958) and Goligher (1958) have suggested that the formation of this space may be avoided by bringing the terminal colon or ileum to the surface through the extraperitoneal tissues. A similar procedure has been used by one of us since 1952, and we feel that our experience over a number of years shows that this method of forming colostomies and ileostomies does not result in any additional immediate or late complications. We therefore consider it worth recording our results in support of the previous writers.

FORMATION OF THE EXTRAPERITONEAL COLOSTOMY

An incision is made in the parietal peritoneum immediately lateral to the lower end of the descending colon. Usually this incision can be a small extension upwards of the incision already made for the mobilization of the sigmoid colon. With a long artery forceps on the edge of the peritoneum, the anterior flap is raised and with two fingers of the right hand a tunnel is easily formed in the retroperitoneal tissues and extended round to the deep surface of the anterior abdominal wall (Fig. 1). A disc of skin 1 in. in diameter is removed from the abdominal wall where the colostomy opening is to be placed, usually at the lateral border of the rectus muscle on a line between the umbilicus and the left anterior superior spine. The muscles deep to the skin opening are incised so as to enter the extraperitoneal tunnel which had just been formed. Long sponge forceps are then passed through the skin opening along the tunnel and so into the peritoneal cavity. The de Martel clamp on the upper end of the previously divided sigmoid colon is grasped by the sponge forceps and is brought out through the extraperitoneal tunnel so that it and a short length of colon lie on the abdominal wall (Fig. 2).
A few catgut stitches are inserted to fix the upper leaf of peritoneum to the colon as it passes extraperitoneally, and in particular to obliterate any small gap that may be present on either side of the colon.

Finally, after the abdomen has been closed, any excess of colon on the skin surface is cut away and the bowel mucosa sutured to the skin edge.

\section{FORMATION OF THE EXTRAPERITONEAL ILEOSTOMY}

The extraperitoneal ileostomy is fashioned in a similar manner by starting the retroperitoneal tunnel in the right iliac fossa just below the lowest point of the small bowel mesentery. The clamped and divided terminal ileum is then drawn through the tunnel on to the skin surface. The same technique is used in bringing an ileal bladder to the skin surface.

\section{RESULTS}

Since 1952, 51 extraperitoneal colostomies and six extraperitoneal ileostomies have been fashioned. Twenty-two of the former have been made in the last 18 months and have functioned without complications. We have reviewed those fashioned before 1959 to see whether any late complications occurred that were attributable to this operation.

EXTRAPERITONEAL COLOSTOMY This operation was performed on 29 patients before 1959; 27 suffered from carcinoma of the rectum, one patient with carcinoma of the bladder was treated by total cystectomy and colostomy with transplantation of the ureters into a rectal bladder, and a further patient with incontinence of faeces due to prolapse of the rectum, which previous surgery had failed to cure, was made comfortable with a left iliac colostomy.

There were three post-operative deaths, and postmortem examination showed these to be due to cerebral thrombosis, salmonella enteritis, and ileus 


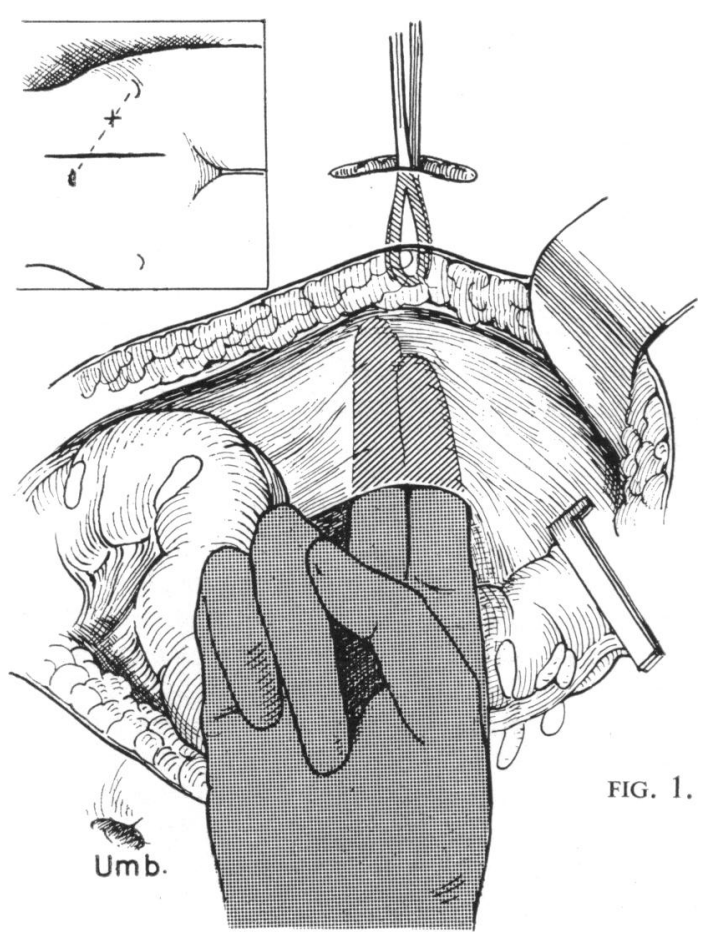

FIG. 1. The formation of the retroperitoneal tunnel.

FIG. 2. Method of passing the crushed and divided bowel through the tunnel.

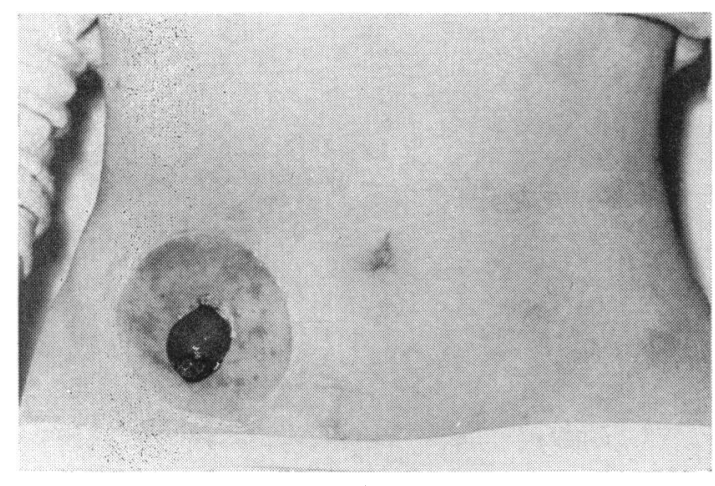

FIG. 3. Ileostomy eight years after operation.

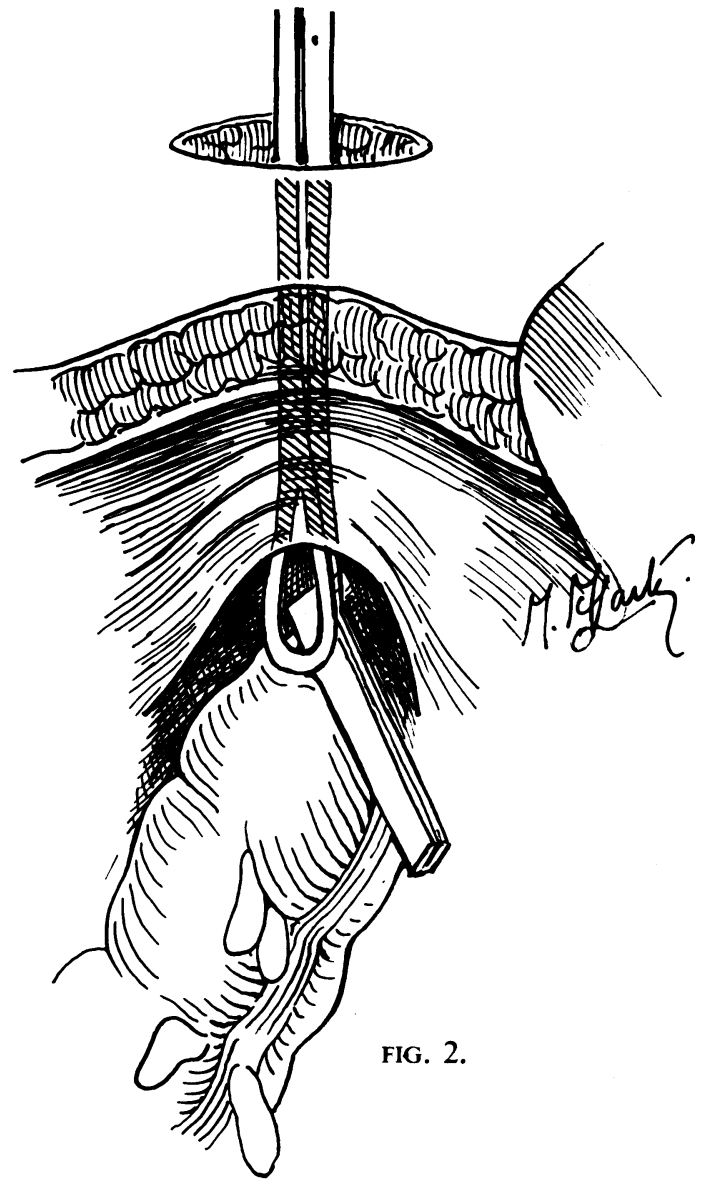

following laparotomy for post-operative obstruction, which was due to adhesions between the small bowel and the pelvic suture line.

Of the remaining 26 patients, 10 are alive and well, 12 have died from their original disease, and four from other causes. Half died in hospital and half at home under the care of their own doctors, who kindly gave us details of their last days.

The average time the colostomies of the deceased patients functioned was over two years. The remainder are still satisfactory after an average of three years and seven months; one of these was fashioned over seven years ago.

Two colostomies became stenosed at the mucocutaneous junction due to scarring. Another was excised en bloc for local recurrence of growth after two years, and replaced by a similar colostomy which functioned for over a year without trouble. In only one case was there prolapse of the mucosa; this was slight and did not require surgery. 
EXTRAPERITONEAL ILEOSTOMY Six patients with ulcerative colitis have been treated by total colectomy. Two of these seriously ill patients died postoperatively and necropsy showed that the ileostomies were uncomplicated. The remaining four patients have been alive for over seven years. One has since had a laparotomy for obstruction due to adhesions, at which 10 in. of gangrenous small bowel was resected. The ileostomy continues to function well, although it has had to be digitally dilated (Fig. 3). There has been no case of prolapse of small bowel through the ileostomy.

\section{DISCUSSION}

Closure of the lateral space may be difficult and if non-absorbable sutures are used there is a risk of a troublesome sinus forming. We have recently seen a case in which an ileostomy was formed and the lateral space closed with catgut, but on a second laparotomy small bowel was found to have prolapsed through the space, which admitted three fingers. This has convinced us that closure of the lateral space with sutures may not be permanent however carefully it is done. The extraperitoneal route does not result in the formation of a lateral space and thus one cause of post-operative obstruction is avoided.

Our experience drawn from this series leads us to believe that the extraperitoneal route of the bowel does not result in any additional complications. Goligher's suggestion that this method may reduce the incidence of prolapse, as the bowel may be better fixed by its oblique exit from the abdomen, is supported by the fact that there has been no case of prolapse requiring surgery in this series. The extraperitoneal tissues do not contract and cause stenosis of the bowel; the only stenosis has been at the skin surface. One ileostomy, complicated by postoperative abscess formation in the surrounding tissues, has functioned without stenosis for over six years. Secondary spread of growth was seen surrounding the last 6 in. of colon in another patient, but the colostomy remained open until the patient's death.

\section{CONCLUSION}

We conclude that the extraperitoneal method of forming a colostomy or an ileostomy is an effective method of avoiding the dangers associated with the formation of a lateral space. The oblique exit of the bowel through the abdominal wall appears to reduce the likelihood of prolapse without resulting in any long-term stenosis of the intramural bowel.

We agree with Sames and Goligher that this operation is satisfactory and have adopted it as our standard technique.

\section{REFERENCES}

Goligher, J. C. (1958). Extraperitoneal colostomy or ileostomy. Brit. J. Surg., 46, 97-103.

Sames, C. P. (1958). Extraperitoneal colostomy. Lancet, 1, 567-568. 